\title{
Somatotipo de Atletas y Practicantes de Gimnasia Rítmica Prepubescentes y Postpubescentes
}

\author{
Somatotype in Rhythmic Gymnastics Athletes and Pre and Post Pubertal Practitioners
}

\author{
Luciana de Souza Menezes; ; Jefferson Novaes ${ }^{* * *}$ \& José Fernandes-Filho**,****
}

\begin{abstract}
MENEZES, L. S.; NOVAES, J. \& FERNANDES-FILHO, J. Somatotipo de atletas y practicantes de gimnasia rítmica prepubescentes y postpubescentes. Int. J. Morphol., 32(3):968-972, 2014.

RESUMEN: El objetivo fue identificar y comparar el somatotipo de atletas y practicantes de gimnasia rítmica de 7 a 25 años en Brasil, divididas en las siguientes categorías: diferentes niveles de competencia (internacional, nacional y regional) y practicantes de la modalidad en la fase prepubescente y postpubescente. El protocolo utilizado para la evaluación fue el somatotipo de Heath \& Carter. Se realizó un estudio descriptivo, de lineación cuasi experimental, donde fueron evaluadas 125 atletas y practicantes de gimnasia rítmica. Para el cálculo de las categorías se aplicó estadística descriptiva por las medidas de posición y dispersión. El análisis de inferencia para las variables fue utilizado a fin de verificar la existencia de diferencias significativas entre los grupos. Fue utilizado el análisis de variancia (ANOVA) con un valor p<0,05, y la prueba de Tuckey. Los resultados para el somatotipo de las diferentes categorías fueron: nivel internacional= ectomorfo balanceado; nivel nacional= ectomorfo balanceado; nivel regional= ectomesomorfo; no menárquicas= endomorfo balanceado y menárquicas= endoectomorfo. Se encontraron diferencias significativas entre los grupos de las variables de endomorfia y mesomorfia. Podemos concluir que las dos categorías más homogéneas en este estudio fueron las de nivel internacional y nacional. La comparación entre la categoría internacional y la categoría no menárquicas fue inversa.
\end{abstract}

PALABRAS CLAVE: Gimnasia; Somatotipo; Menarquia.

\section{INTRODUCCIÓN}

La gimnasia rítmica se clasificó como uno de los deportes más atractivos, debido a su apelación artística, ya que fue utilizada en los Juegos Olímpicos de 1984 y en los Juegos Olímpicos de Los Ángeles (Kwitniewska et al., 2009). El creciente desarrollo de las manifestaciones rítmicas en Brasil, y la reciente conquista de títulos inéditos, como el tetracampeonato de Brasil en los juegos panamericanos en la ciudad de Guadalajara el año 2011, generaron la necesidad de obtener más informaciones sobre los aspectos relacionados a los atletas y a los practicantes de esta modalidad. La carencia de informaciones acerca de las características físicas de las practicantes y atletas de esa modalidad así como la necesidad del fomento a nuevas investigaciones en ese deporte en especial en atletas de América Latina.

Esta modalidad presenta atletas de alto rendimiento que alcanzan el estatuto internacional antes de convertirse en adultos. Durante la adolescencia, estos atletas participan de programas de capacitación intensa durante el período de grandes cambios físicos y hormonales (Menezes et al., 2009). De acuerdo con Poliszczuk \& Broda (2010), la gimnasia rítmica se caracteriza por el biotipo de quien la practica. El conocimiento de estas características propicia la aplicación adecuada de las estrategias que influencian en su mejor rendimiento. En relación a la idea expuesta anteriormente, desde el punto de vista la eficacia, el biotipo específico y los patrones corporales de las gimnastas favorecen la ejecución de los movimientos técnicos específicos de esta modalidad (Amigó et al., 2009; Miletic et al., 2004; Vieira et al., 2009).

El somatotipo, la composición corporal y la maduración psicológica son de interés para miembros de los programas existentes en la identificación y selección de talentos deportivos (Avila et al., 2012; Cuk et al., 2012; Herm, 2010; Fonseca \& Fernandes Filho, 2010, Fonseca et al., 2008; Linhares et al., 2009; Santos et al., 2008; Zary \& Fernandes Filho, 2007; Zary et al. 2010). A partir de los presupuestos presentados, este estudio propone identificar

\footnotetext{
* Universidade de Trás-os-Montes e Alto D`ouro-UTAD, Vila Real, Portugal.

** Universidade Federal do Rio de Janeiro-UFRJ, Rio de Janeiro, Brasil.

**** Laboratório de Biociências do Movimento Humano LABIMH/UFRJ, Rio de Janeiro, Brasil.
} 
y comparar el somatotipo y la constitución corporal de las deportistas y practicantes de gimnasia rítmica, entre 7 y 25 años, en Brasil. Para esto, el grupo de estudio fue dividido en las siguientes categorías: diferentes niveles competitivos (internacional, nacional y regional) y practicantes de la modalidad en etapas prepubescente (no menárquicas) y postpubescente (menárquicas).

\section{MATERIAL Y MÉTODO}

Este estudio, siguió las normas internacionales para la experimentación con seres humanos de la declaración de Helsinki de 1975 y la Resolución 196-1996 del Consejo Nacional de Salud de Brasil. Se desarrolló un estudio descriptivo de carácter transversal, con tipología y delineación comparativa. En concordancia con esta tipología, fue utilizada la comparación común y de las variables en las diferentes categorías, determinándose que las diferencias no pueden ser atribuidas al azar. El tratamiento estadístico sólo determinó si los grupos son diferentes, sin embargo no mostró el motivo de la diferencia de los grupos, por lo tanto, el objetivo es la aceptación o el rechazo de la hipótesis nula (Thomas et al., 2007).

Se incluyeron en el grupo de estudio a las atletas, federadas, y practicantes de gimnasia rítmica, con edades entre 7 y 25 años, todas del sexo femenino. Este grupo fue compuesto por 125 gimnastas, de acuerdo a las siguientes categorías: a) nivel internacional: compuesto por 8 gimnastas con edad promedio de 17,4 $\pm 3,4$, integrantes de la Selección Brasileña; b) nivel nacional: compuesto por 10 atletas con edad promedio de $13,7 \pm 1,3$, participantes del Campeonato Brasileño adulto y c) nivel provincia/regional: compuesto por 7 gimnastas con edad promedio de 13,9 $\pm 1,3$ participantes del Campeonato Provincial de Rio de Janeiro, en la categoría de adultos, 100 practicantes de la modalidad en el estado de Rio de Janeiro, dividida entre

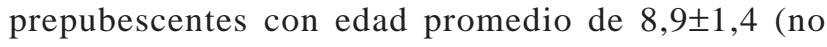
menárquicas) y postpubescentes con edad promedio de $14,1 \pm 2,0$ (menárquicas). Fueron evaluadas 49 gimnastas que vivenciaron la menarquia y 51 gimnastas que no.

El protocolo utilizado para la determinación de la función del somatotipo siguió el método somatotipológico de Heath \& Carter (ISAK, 2000).

Para determinar el peso corporal se utilizó una Balanza Filizola (São Paulo, Brasil), debidamente calibrada, con una precisión de $100 \mathrm{~g}$ y una escala variante de 0 a $150 \mathrm{Kg}$. Para medir la estatura, se utilizó un Estadiómetro con un plano vertical $(210 \mathrm{~cm})$ conectado en ángulo recto a un plano horizontal $(40 \mathrm{~cm})$, con una escala vertical de base, métrica, graduada en centímetros y pulgadas, junto a un cursor $(10 \mathrm{~cm})$ que desliza sobre el plano vertical. Para evaluar las medidas se utilizó la cinta métrica periférica de metal flexible (Sanny), con $150 \mathrm{~cm}$ de longitud (precisión de $0,1 \mathrm{~cm}$ ). Los pliegues cutáneos fueron medidos con un compás de pliegues cutáneos Harpender (precisión de 0,1 mm). Para medir los diámetros óseos se utilizó un Paquímetro Rosscraft (Modelo Tommy2, Canadá), presentando una variación entre 2 y $16 \mathrm{~cm}$ y una graduación de $1 \mathrm{~mm}$.

Para estudiar las categorías, fue utilizada la estadística descriptiva con el cálculo de las medidas de posición y dispersión. Fueron calculadas la media y medianas, desviación estándar, y la medida de dispersión que comprueba la variabilidad existente entre los datos alrededor de la media. En relación a las variables continuas, fue utilizado el análisis inferencial para comprobar la existencia de diferencias estadísticamente significativas entre los grupos de las practicantes menárquicas y no menárquicas, atletas de la provincia, atletas nacionales y atletas de nivel internacional (Selección Brasileña). Para eso fue utilizado el análisis de variancia (ANOVA), y para definir la significancia de la prueba, se utilizó un valor $\mathrm{p}<0,05$. Cuando se compró existencia de diferencia estadística significativa, fue utilizada la prueba de Tukey para identificar el grupo distinto de los demás, con un nivel de significación a=5,0\% .

\section{RESULTADOS}

En la Tabla I se presentan los resultados estadísticos descriptivos (media, desviación estándar, mínimo y máximo) de las variables edad, altura y peso de los sujetos de la muestra evaluada, mientras que en la Tabla II, estos valores corresponden a las variables de endomorfia, mesomorfiay ectomorfia.

Para analizar la diferencia entre los promedios de las calificaciones corporales (endomorfia, mesomorfia y ectomorfia) en las categorías de estudio, se utilizó el análisis de variancia ANOVA y, a continuación, la prueba Post Hoc de Tukey. La Tabla III muestra que la categoría de clasificación endomorfia-menárquicas comparada a la categoría no menárquicas, regionales, nacionales e internacionales, tuvo una diferencia significativa $\left(\mathrm{p}<0,05^{* *}\right)$. Lo mismo puede decirse de la categoría no menárquica en relación a los niveles regionales, nacionales e internacionales. Se observó, sin embargo, que entre las categorías competitivas (estatal, nacional e internacional) hubo diferencias estadísticamente significativas. 
La Tabla IV presenta los resultados de la clasificación de mesomorfia, y muestra que no hubo ninguna diferencia estadísticamente significativa al comparar menárquicas con no menárquicas ( $\mathrm{p}<0,01 * *)$. En otras categorías no fueron registrados resultados con diferencias estadísticamente significativas.
En la clasificación de ectomorfia no fue observada ninguna diferencia estadísticamente significativa en las comparaciones entre las categorías según la prueba de Tuckey (Tabla V). Los resultados muestran la existencia de variación en cada categoría, pero la variación entre las categorías calculadas por ANOVA $(\mathrm{p}=0,017)$.

Tabla I. Resultados descriptivos de la edad de las variables, peso y estatura.

\begin{tabular}{|c|c|c|c|c|c|c|c|c|c|c|}
\hline \multirow{2}{*}{ Categorías } & \multirow{2}{*}{$\mathbf{n}$} & \multicolumn{3}{|c|}{ Edad } & \multicolumn{3}{|c|}{ Estatura } & \multicolumn{3}{|c|}{ Peso } \\
\hline & & Media $\pm D E$ & Mínimo & Máximo & Media $\pm D E$ & Mínimo & Máximo & Media $\pm D E$ & Mínimo & Máximo \\
\hline Menárquicas & 49 & $14,1 \pm 2,0$ & 11 & 19 & $158,0 \pm 5,80$ & 149,0 & 176,0 & $47,1 \pm 8,22$ & 35,0 & 70,0 \\
\hline No menárquicas & 51 & $8,9 \pm 1,4$ & 7 & 11 & $137,7 \pm 10,91$ & 121,0 & 166,0 & $33,4 \pm 9,00$ & 20,0 & 59,0 \\
\hline Regional & 7 & $13,9 \pm 1,3$ & 12 & 16 & $155,0 \pm 0,03$ & 152,0 & 159,0 & $42,1 \pm 2,60$ & 39,3 & 47,0 \\
\hline Nacional & 10 & $13,7 \pm 1,3$ & 12 & 16 & $156,0 \pm 0,03$ & 152,0 & 163,0 & $43,1 \pm 3,86$ & 35,5 & 48,0 \\
\hline Internacional & 8 & $17,4 \pm 3,4$ & 14 & 25 & $162,0 \pm 0,06$ & 155,5 & 176,0 & $47,8 \pm 6,27$ & 42,4 & 60,6 \\
\hline
\end{tabular}

Tabla II. Clasificación Somatotípica.

\begin{tabular}{lcccc}
\hline \multirow{2}{*}{ Categorías } & \multicolumn{3}{c}{ Media \pm DE } \\
\cline { 2 - 5 } & Endomorfia & Mesomorfia & Ectomorfia & Clasificación \\
\hline Menárquica s & $4,20 \pm 1,47$ & $2,67 \pm 0,95$ & $3,72 \pm 1,37$ & Endoectomorfo \\
No menenárquicas & $5,42 \pm 0,96$ & $3,31 \pm 0,97$ & $3,03 \pm 1,41$ & Endomorfo Balanceado \\
Regional & $2,88 \pm 0,82$ & $3,16 \pm 0,40$ & $3,51 \pm 0,63$ & Ectomesomorfo \\
Nacional & $2,70 \pm 0,99$ & $2,65 \pm 0,87$ & $4,17 \pm 0,92$ & Ectomorfo Balanceado \\
Internacional & $2,30 \pm 0,38$ & $2,86 \pm 0,37$ & $4,16 \pm 0,64$ & Ectomorfo Balanceado \\
\hline
\end{tabular}

Tabla III. Resultado del Post Hoc de Tuckey para endomorfia.

\begin{tabular}{lc}
\hline Prueba de comparación múltiple de Tukey & Valor p \\
\hline Menárquicas x No menárquicas & $\mathrm{p}<0,001^{*}$ \\
Menárquicas x Regional & $\mathrm{p}>0,05^{*}$ \\
Menárquicas x Nacional & $\mathrm{p}<0,001^{*}$ \\
Menárquicas x Internacional & $\mathrm{p}<0,001^{*}$ \\
No menárquicas x Regional & $\mathrm{p}<0,001^{*}$ \\
No menárquicas x Nacional & $\mathrm{p}<0,001^{*}$ \\
No menárquicas x Internacional & $\mathrm{p}<0,001^{*}$ \\
Regional x Nacional & $\mathrm{p}>0,05$ \\
Regional x Internacional & $\mathrm{p}>0,05$ \\
Nacional x Internacional & $\mathrm{p}>0,05$ \\
\hline
\end{tabular}

Tabla IV. Resultado del Post Hoc de Tuckey para mesomorfia.

\begin{tabular}{ll}
\hline Prueba de comparación múltiple de Tukey & Valor p \\
\hline Menárquicas x No menárquicas & $\mathrm{p}<0,01^{*}$ \\
Menárquicas x Regional & $\mathrm{p}>0,05$ \\
Menárquicas x Nacional & $\mathrm{p}>0,05$ \\
Menárquicas x Internacional & $\mathrm{p}>0,05$ \\
No menárquicas x Regional & $\mathrm{p}>0,05$ \\
No menárquicas x Nacional & $\mathrm{p}>0,05$ \\
No menárquicas x Internacional & $\mathrm{p}>0,05$ \\
Regional x Nacional & $\mathrm{p}>0,05$ \\
Regional x Interna cional & $\mathrm{p}>0,05$ \\
Nacional x Interna cional & $\mathrm{p}>0,05$ \\
\hline
\end{tabular}

Tabla V. Resultado del Post Hoc de Tuckey para ectomorfia.

\begin{tabular}{ll}
\hline Prueba de comparación múltiple de Tukey & Valor p \\
\hline Menárquicas x No menárquicas & $\mathrm{p}>0,05$ \\
Menárquicas x Regional & $\mathrm{p}>0,05$ \\
Menárquicas x Nacional & $\mathrm{p}>0,05$ \\
Menárquicas x Internacional & $\mathrm{p}>0,05$ \\
No menárquicas x Regional & $\mathrm{p}>0,05$ \\
No menárquicas x Nacional & $\mathrm{p}>0,05$ \\
No menárquicas x Internacional & $\mathrm{p}>0,05$ \\
Regional x Nacional & $\mathrm{p}>0,05$ \\
Regional x Internacional & $\mathrm{p}>0,05$ \\
Nacional x Internacional & $\mathrm{p}>0,05$ \\
\hline
\end{tabular}

\section{DISCUSIÓN}

Basado en los valores anteriores uno puede clasificar el somatotipo y la constitución corporal de las diferentes categorías buscado como: nivel internacional= ectomorfo balanceado; nivel nacional= ectomorfo balanceado; nivel regional $=$ ectomesomorfo; no menárquicas $=$ endomorfo balanceado y menárquicas $=$ endoectomorfo.

De acuerdo con autores como Poliszczuk \& Broda, Amigó et al. y Broda \& Poliszczuk (2009), la literatura su- 
giere que la prevalencia de somatotipo y la constitución corporal de gimnastas de alto rendimiento presentan un perfil ectomorfo corroborando con los resultados encontrados en este estudio, ya que el componente ectomórfico y el nivel competitivo aumenta visiblemente. Sin embargo, con el componente endomórfico sucede a la inversa.

En relación a la investigación realizada por Menezes et al., resultados similares fueron encontrados para este estudio, sobre los valores de cada componente del somatotipo y la constitución corporal que se encuentran en diferentes niveles competitivos de gimnastas. El somatotipo y componentes de constitución corporal presentan los siguientes valores de Media $\pm D E$ : endomorfia: selección brasileña= $2,33 \pm 0,40$; nivel nacional $=2,48 \pm 1,02$ y nivel regional $=$ 2,88 $\pm 0,82$; mesomorfia: selección brasileña $=2,83 \pm 0,39$; nivel nacional $=2,81 \pm 1,00$ y nivel regional $=3,16 \pm 0,40$; ectomorfia: selección brasileña $=4,17 \pm 0,69$; nivel nacional $=$ $4,06 \pm 1,05$ y nivel regional $=3,51 \pm 0,63$.

En el estudio de Menezes et al., hecho con gimnastas federadas, fue observado el predominio del componente ectomórfico. Ellos agregan que fueron encontrados resultados semejantes a los resultados del estudio presentado, en relación a los valores medios de cada componente del somatotipo encontrados en las gimnastas de diferentes niveles competitivos.

Para las comparaciones entre grupos, no se encontró, en este estudio, ninguna diferencia significativa en las comparaciones entre las categorías competitivas para ninguno de los componentes del somatotipo y de la constitución corporal. Tales diferencias fueron percibidas en las comparaciones con categorías menárquicas x no menárquicas $\left(\mathrm{p}<0.01^{*}\right)$ en el componente mesomórfico y en las comparaciones con las practicantes en la endomorfia de las siguientes categorías: menárquicas $\mathrm{x}$ no menárquicas $(\mathrm{p}<0,001 *)$, estado de menárquicas $\mathrm{x}\left(\mathrm{p}<0,05^{*}\right)$, menárquicas $\mathrm{x}$ nacional $(\mathrm{p}<0,01 *)$, menárquicas $x$ internacional $(\mathrm{p}<0,001 *)$, no regional $\mathrm{x}$ menárquicas $(\mathrm{p}<0,001 *)$, no menárquicas $\mathrm{x}$ nacional $\left(\mathrm{p}<0,001^{*}\right)$ y no menárquicas $\mathrm{x}$ internacional $(\mathrm{p}<0,001 *)$. Estos resultados son semejantes a los reportados por Amigó et al., quienes comparan a las gimnastas del mismo nivel técnico de diferentes edades. También es semejante al estudio de Menezes \& Fernandes Filho (2006), ya que afirman que hay diferencias estadísticas significativas en el somatotipo y en la constitución corporal entre los distintos niveles de categorías competitivas.

MENEZES, L. S.; NOVAES, J. \& FERNANDES-FILHO, J. Somatotype in Rhythmic Gymnastics Athletes and pre and post pubertal practitioners. Int. J. Morphol., 32(3):968-972, 2014.

SUMMARY: The objective of the present study was to identify and compare the somatotype of 125 Brazilian athletes and practitioners of Rhythmic Gymnastics of different qualification levels from 7 to 25 years old, divided into the following categories: different competitive levels (international, national and regional) and pre and post menarche practitioners. One hundred twenty-five Brazilian athletes and practitioners of Rhythmic Gymnastics of different levels of qualification from 7 to 25 years old were evaluated. The protocol used was Heath \& Carter somatotype. The descriptive study was applied. In order to define the profile of the data collected, the descriptive statistics with measurements of position and dispersion were estimated. The Analysis of Variance was applied. Inferential analysis, for continuous variables, was used in order to identify the existence of significant statistical differences among the Post menarche, Pre menarche, international level, national level and regional level. Afterwards the Post Hoc Tuckey test was applied. According to the results, the somatotype for the different categories are: international level= balanced ectomorphy, national= balanced ectomorphy, regional= ectomesomorphy, pre menarche practitioners $=$ balanced endomorphy and post menarche practitioners= endoectomorphy. Significant statistical differences were found in the following variables: endomorphy and mesomorphy. It is concluded that the most similar categories in the present study were the national and regional levels, on the other hand, the most different categories were the pre menarcheal and the international level.

KEY WORDS: Gymnastics; Somatotype; Menarche.

\section{REFERENCIAS BIBLIOGRÁFICAS}

Amigó, A. I.; Faciabén, A. B.; Evrard, M. M.; Ballarini, P. A. G. \& Marginet, M. C. Talla, peso, somatotipo y composición corporal en gimnastas de elite españoles desde la infancia hasta la edad adult. Apunts Med. Esport, 161(44):18-28, 2009.

Avila, C. B.; Cea, B. M. C.; Padilla, E. L. \& de la Rosa, F. J. B. Body Composition and Somatotype in Women's Football.
South American Championship Sub-17. Int. J. Morphol., 30(2):450-60, 2012.

Broda, D. \& Poliszczuk, T. The dynamic balance and body build types of girls aged 8-11 years old who practice rhythmic gymnastics. In: Mezinárodní studentská ve`decká konference, Univerzita Karlova v Praze, Fakulta te`lesné vłchovy a sportu. 
Praha, Univerzita Karlova v Praze, Fakulta te`lesné vłchovy a sportu, 2009.

Cuk, I.; Pajek, M. B.; Jakse, B.; Pajek, J. \& Pecek, M. Morphologic Bilateral Differences of Top Level Gymnasts. Int. J. Morphol., 30(1):110-4, 2012.

Fonseca, C. \& Fernandes Filho, J. Características dermatoglíficas de atletas de voleibol masculino sub 17 do Brasil em diferentes níveis de qualificação esportiva. Gymn. Rev. Educ. Fís. Desporto Saúde, 1(2):115-36, 2010.

Fonseca, C. L. T.; Dantas, P. M. S.; Fernandes, P. R. \& Fernandes Filho, J. Dermatoglyphic, somatotype, and explosive strength profiles of women's volleyball of the Brazilian team. Fit. Perform. J., 7(1):35-40, 2008.

Herm, K. P. Aspects of cooperation in kinanthropometry between India and Germany and an example of different ways of talent identification and selection. Br. J. Sports Med., 44 (Suppl. 1):i32, 2010 .

International Society of Advance of the Kinesiantropometric (ISAK). Apostila de curso. Rio de Janeiro, International Society of Advance of the Kinesiantropometric (ISAK), 2000.

Kwitniewska, A.; Dornowski, M. \& Hökelmann, A. Quantitative and qualitative analysis of international standing in group competition in the sport of rhythmic gymnastics. Baltic J. Health Phys. Act., 1(2):118-25, 2009.

Linhares, R. V.; Matta, M. O.; Lima, J. R.; Dantas, P. M.; Costa, M. B. \& Fernandes Filho, J. Effects of sexual maturation on body composition, dermatoglyphics, somatotype and basic physical qualities of adolescents. Arq. Bras. Endocrinol. Metabol., 53(1):47-54, 2009.

Menezes, L.; Dantas, P. \& Fernandes Filho, J. Rhythmic Gymnastics On Different Levels Of Qualification. A Comparative Study. In: American College of Sports Medicine - Annual Meeting, 2009. Seattle, Medicine and Science in Sports and Exercise, 2009.

Menezes, L. \& Fernandes Filho, J. Identificação e comparação das características dermatoglíficas, somatotípicas e de qualidades físicas básicas de atletas de GRD de diferentes níveis de qualificação esportiva. Fit. Perform. J., 5(6):393-401, 2006.

Miletic, D.; Katic, R. \& Males, B. Some anthropologic factors of performance in rhythmic gymnastics novices. Coll. Antropol., 28(2):727-37, 2004.

Poliszczuk, T. \& Broda, D. Somatic constitution and the ability to maintain dynamic body equilibrium in girls practicing rhythmic gymnastics. Pediatr. Endocrinol. Diabetes Metab., 16(2):94-9, 2010.

Santos, L. C.; Dantas, P. M. \& Filho, J.F. Características genotípicas e fenotípicas em atletas velocistas. Motricidade, 4(1):49-56, 2008.
Thomas, J. R.; Nelson, J. K. \& Silvermam, S. J. Métodos de pesquisa em atividade física. $5^{\mathrm{a}}$ ed. Porto Alegre, Artmed, 2007.

Vieira, J. L.; Amorim, H. Z.; Vieira, L. F.; Amorim, A. C. \& Rocha, P. G. Distúrbios de atitudes alimentares e distorção da imagem corporal no contexto competitivo da ginástica rítmica. Rev. Bras. Med. Esporte, 15(6):410-14, 2009.

Zary, J. C.; Reis, V. M.; Rouboa, A.; Silva, A. J.; Fernandes, P. R. \& Filho, J. F. The somatotype and dermatoglyphic profiles of adult, junior and juvenile male Brazilian top-level volleyball players. Sci. Sports, 25(3):146-52, 2010.

Zary, J. C. \& Fernandes Filho, J. Identificação do Perfil dermatoglífico e somatotípico dos atletas de voleibol. Masculino adulto, Juvenil e infanto-Juvenil, de alto Rendimento no Brasil. Rev. Bras. Ciênc. Mov., 15(1):53-60, 2007.

\author{
Dirección para Correspondencia: \\ Luciana Menezes, M. Sc. \\ Universidade de Trás-os-Montes e Alto D`ouro-UTAD \\ Vila Real \\ PORTUGAL
}

Emai: lucianasmenezes@hotmail.com

Recibido: 01-08-2012

Aceptado: 31-07-2014 